\title{
Clinical, electrophysiological, and molecular genetic studies in a new family with paramyotonia congenita
}

\author{
N P Davies, L H Eunson, R P Gregory, K R Mills, P J Morrison, M G Hanna
}

\begin{abstract}
Objectives-To characterise the clinical and electrophysiological features and to determine the molecular genetic basis of pure paramyotonia congenita in a previously unreported large Irish kindred.

Methods-Clinical and neurophysiological examination was performed on three of the five affected family members. Five unaffected and three affected members of the family were available for genetic testing. Direct sequence analysis of the SCN4A gene on chromosome $17 q$, was performed on the proband's DNA. Restriction fragment length polymorphism

(RFLP) analysis was used to screen other family members and control chromosomes for the SCN4A mutation identified. Results-Each affected member had clinical and examination features consistent with pure paramyotonia congenita. Electrophysiological studies disclosed a $78 \%$ drop in compound muscle action potential (CMAP) amplitude on cooling to $20^{\circ} \mathrm{C}$. DNA sequence analysis identified a heterozygous point mutation G4367A in exon 24 of the SCN4A gene which segregated with paramyotonia and was absent in 200 control chromosomes. The mutation is predicted to result in a radical amino acid substitution at a highly conserved position within the voltage sensing fourth transmembrane segment of the fourth repeated domain of the sodium channel.
\end{abstract}

University

Department of Clinical Neurology, Institute of Neurology, Queen Square, London WC1N 3BG, UK

N P Davies

L H Eunson

M G Hanna

The Battle Hospital, Oxford Road, Reading, UK

R P Gregory

King's Neuroscience Centre, King's College Hospital, Denmark Hill, London, UK K R Mills

Northern Ireland Regional Genetics Centre, Belfast City Hospital Trust,

Lisburn Road, Belfast, Northern Ireland, UK

P J Morrison

Correspondence to: Dr MG Hanna,

Neurogenetics and Muscle Sections, University

Department of Clinical

Neurology, Institute of

Neurology, Queen Square,

London. UK. WC1N 3BG,

UK

emailmhanna@ion.ucl.ac.uk

Received 3 August 1999 and in revised form

2 November 1999

Accepted 11 November 1999
Conclusions-The G4367A mutation is likely to be pathogenic and it associates with a pure paramyotonia phenotype. In keeping with other paramyotonia mutations in this region of the skeletal muscle sodium channel, it is predicted that this mutation will impair voltage sensing or sodium channel fast inactivation in a temperature dependent fashion. This study provides further evidence that exon 24 in SCN4A is a hot spot for paramyotonia mutations and this has implications for a DNA based diagnostic service.

(F Neurol Neurosurg Psychiatry 2000;68:504-507)

Keywords: paramyotonia congenita; sodium channel; channelopathy
Skeletal muscle channelopathies are disorders of muscle fibre membrane excitability and present clinically with varying combinations of periodic paralysis, myotonia, and paramyotonia. ${ }^{1}$ Mutations in the skeletal muscle voltage gated sodium channel gene (SCN4A) associate with three clinically distinct disorders; paramyotonia, hyperkalaemic periodic paralysis, and potassium aggravated myotonia. However, the relation between genotype and phenotype in these sodium channel diseases is not always clear cut. ${ }^{2-4}$ We have studied the clinical and electrophysiological features of a previously unreported large Irish pedigree with paramyotonia. Molecular genetic analysis of this family has provided additional evidence that the pure paramyotonia phenotype is particularly associated with point mutations in exon 24 of SCN4A.

\section{Patients and methods}

PATIENTS

The proband was a 27 year old man who had developed symptoms in early childhood. He described "spasms" of muscle stiffness that preferentially affected the hands and face. $\mathrm{He}$ had not noticed any difficulty in swallowing and did not experience stiffness in the lower limbs. There was no "warm up" phenomenon and the symptoms tended to worsen with exertion (paradoxical myotonia). The most marked exacerbation occurred in cold weather. When outside in the winter months, he noticed that his face would "stick" if he smiled and that he was unable to open his hands. They would feel weak until he returned to a warm environment. There was no history of periodic paralysis or progressive weakness. Examination disclosed paradoxical myotonia of grip and eyelid closure but no lid lag. There was percussion myotonia of the thenar eminence. Muscle hypertrophy and weakness were absent. Treatment with low dose mexiletine during the winter was sufficient to relieve symptoms. Four members of his family had a clinical history suggestive of paramyotonia without periodic paralysis. The proband's son, sister, and mother also demonstrated typical signs of paramyotonia on examination (his son was more severely affected, fig 1). 


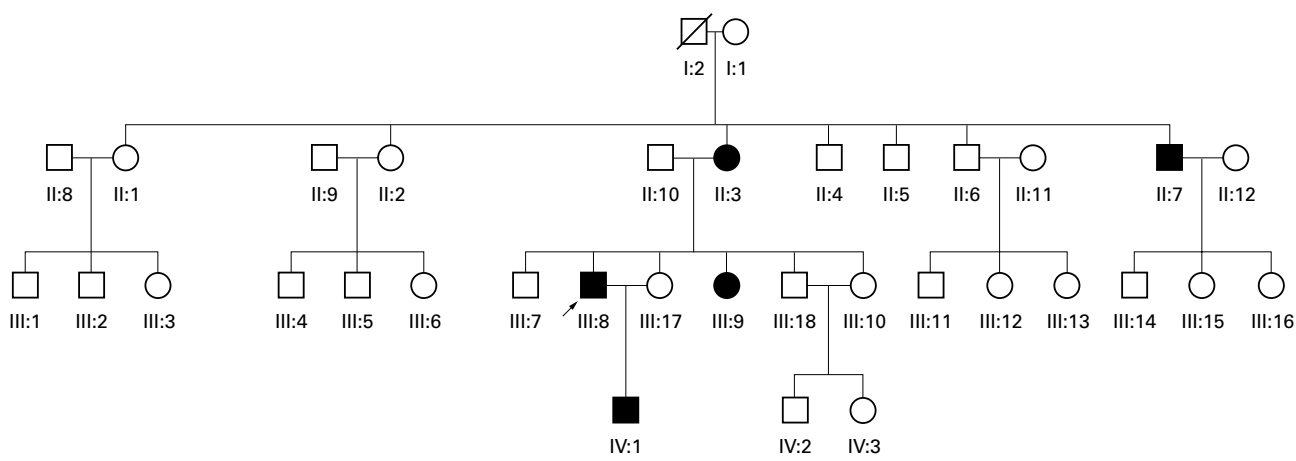

Figure 1 Pedigree of Irish family with paramyotonia congenita demonstrating an autosomal dominant pattern of inheritance. Filled symbols represent affected members, circles for females and squares for males. Filled arrow denotes the proband.

METHODS

Neurophysiology

Routine nerve conduction studies and electromyography were performed. In addition, the protocol reported by Streib for the short exercise test with muscle cooling was followed. ${ }^{5}$

\section{Molecular genetics}

Three affected and five unaffected members of the kindred were available for genetic testing and gave informed consent (fig $2 \mathrm{~A}$ ). Extraction of DNA from blood samples was performed using standard methods. Direct sequence analysis of the SCN4A gene in the proband (III-8) was performed. Oligonucle- otide primers tagged with M13 tails were employed. The sequence of the forward primer was: 5'-TCCCTCGCAGGCCTTGCC-3' and the reverse primer: 5'-GGCGTCGGGGTCGAACTTCT-3'. The polymerase chain reaction (PCR) conditions were as follows: an initial denaturing step at $94^{\circ} \mathrm{C}$ for 3 minutes followed by 30 cycles of the following: $94^{\circ} \mathrm{C}$ for 30 seconds, $60^{\circ} \mathrm{C}$ for 30 seconds, and $72^{\circ} \mathrm{C}$ for 30 seconds. A final extension step of $72^{\circ} \mathrm{C}$ for 10 minutes was used. The products were cleaned using QIAGEN PCR purification kits and both strands were sequenced using a Dye Primer Taq cycle sequencing kit (ABI Applied Biosystems). The sequencing products were
A
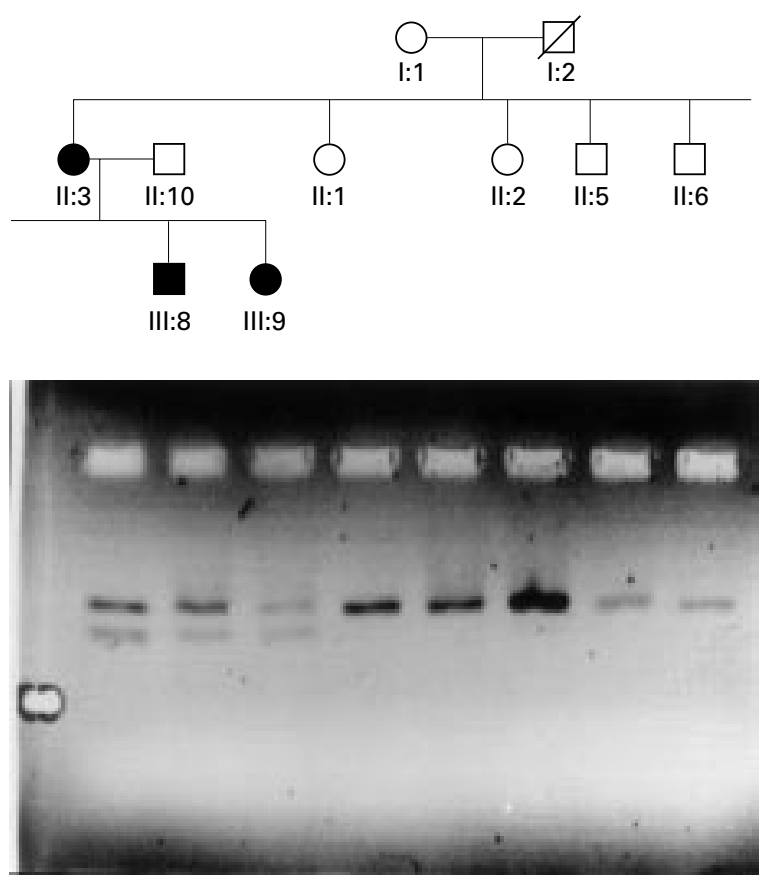

B

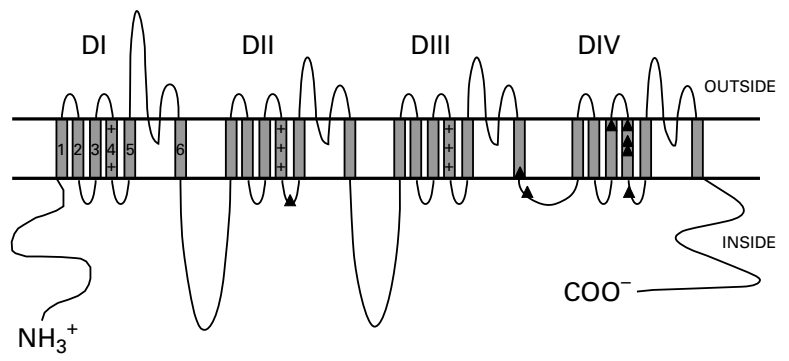

C

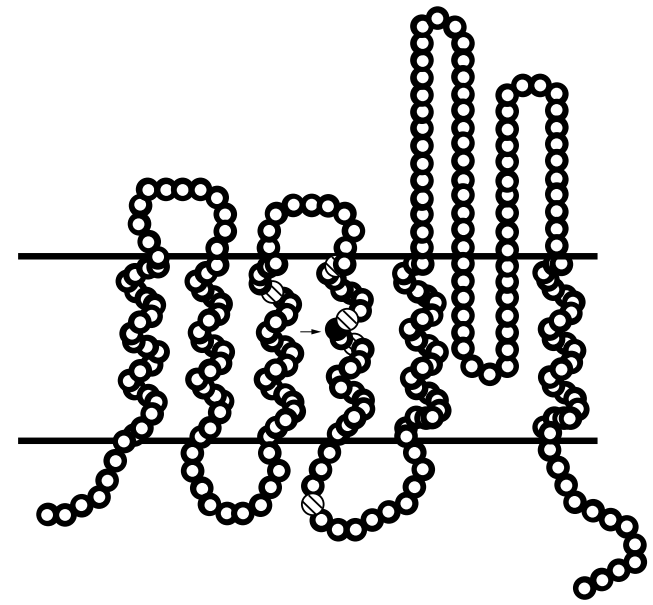

Figure 2 (A) Upper panel shows the members of the pedigree available for genetic analysis. Lower panel shows an agarose gel containing the DNA fragments from the corresponding members of the pedigree after digestion with the restriction enzyme DdeI. The upper band is the undigested $119 \mathrm{bp}$ fragment and the lower band is the digested $90 \mathrm{bp}$ fragment. The $29 \mathrm{bp}$ fragment has migrated off the gel. Unaffected members have a single $119 \mathrm{bp}$ fragment, whereas affected members (II:3, III:8, III:9) are heterozygous for the mutation and have two bands (119 bp and $90 \mathrm{bp})$. (B) A schematic representation of the a subunit of the skeletal muscle voltage gated sodium channel. The repeated domains (DI-DIV) each with six transmembrane helices (S1-S6) are shown. The filled triangles indicate the site of mutations causing paramyotonia within the channel. These concentrate within domain four and particularly in the voltage sensing segment (S4). (C) A magnified view of domain IV from (B). Each sphere represents a single amino acid. The G1456E mutation is shown as a filled sphere within the voltage sensing segment (S4). The striped spheres indicate the sites of mutations causing paramyotonia that occur in exon 24 of the SCN $4 A$ gene. 
run on a $6 \%$ polyacrylamide-urea gel in a $373 \mathrm{~A}$ automated DNA sequencer (ABI). The sequences were analysed using Sequencing Analysis and AutoAssembler software (ABI).

RFLP analysis was then performed to screen control chromosomes and other family members for the mutation identified. As the point mutation discovered neither created nor destroyed a natural restriction site, a mismatch PCR technique was employed. The sequence of the forward (mismatch) primer was: 5'CTGTTCCGTGTGATCCGCCTGGCGCGGACT-3' (mismatch nucleotide shown bold and underlined) and the reverse primer was: 5'-GATGTTGAAGAGGGCAGGCA-3'. The PCR conditions were the same as above, except an annealing temperature of $55^{\circ} \mathrm{C}$ was used. The wild type sequence between nucleotide positions 4360 and 4368 is CGGATTGGG. After amplification with the mismatch primers, the following sequence is generated for the wild type: CGGACTGGG. In the presence of the mutant, the sequence CGGACTGAG is generated. The restriction enzyme DdeI contains the recognition site $\mathrm{C} / \mathrm{TNAG}$, so the mutant sequence is recognised and cut, and the wild type sequence is not cut. After digestion with $D d e I$ according to the manufacturers recommendations, the fragments were separated on a $4 \%$ agarose gel stained with ethidium bromide and visualised on an ultraviolet light box. The $119 \mathrm{bp}$ product was digested into two fragments in the mutant, of 90 and 29 base pairs. The lower panel in figure 2 A shows the products of digestion for members in the pedigree. The upper band is the undigested $119 \mathrm{bp}$ fragment and the lower band is the digested $90 \mathrm{bp}$ fragment. The $29 \mathrm{bp}$ fragment has migrated off the gel. Unaffected members have a single 119 bp fragment, whereas affected members are heterozygous for the mutation and have two bands (119 bp and $90 \mathrm{bp}$ ).

\section{Results}

NEUROPHYSIOLOGY

Nerve conduction studies were normal but EMG showed profuse myotonic discharges. Baseline CMAP was $11.0 \mathrm{mV}$ at a skin temperature of $30^{\circ} \mathrm{C}$. The abductor digiti minimi was then exercised isometrically for 10 seconds. Postexercise supramaximal stimuation elicited a CMAP of $11.3 \mathrm{mV}$. After cooling the arm to $20^{\circ} \mathrm{C}$ and then rewarming, the CMAP dropped to $2.4 \mathrm{mV}$ (78\% decrease) confirming the clinical diagnosis of paramyotonia congenita. Nerve conduction studies in the proband's mother and sister (fig 1 III: 9 and II:3) were normal but EMG demonstrated prominent myotonic discharges.

\section{MOLECULAR GENETICS}

A heterozygous point mutation was identified at nucleotide position 4367 ( $\mathrm{G}$ to $\mathrm{A}$ ). This predicted an amino acid change from glycine to glutamic acid at position 1456 in segment four of the fourth repeated domain of the $\alpha$ subunit of the skeletal muscle sodium channel (G1456E). This glycine is known to be highly conserved through evolution (fig $2 \mathrm{~B}$ and $\mathrm{C}$ ).

\section{Discussion}

The point mutation we have identified in this large Irish kindred with paramyotonia exhibits characteristics which suggest it is the cause of the disease in this family. Firstly, it occurs at a position in exon 24 of the SCN4A gene which is highly conserved suggesting functional importance. Secondly, it is absent from a large panel of 200 control chromosomes indicating that it is unlikely to represent a neutral polymorphism. Thirdly, it segregates with the paramyotonia phenotype in this family. Furthermore, this study confirms a recent report in which the same mutation was identified in a Japanese family with a pure paramyotonia phenotype. ${ }^{6}$ Taken together these findings strongly suggest that the G4367A mutation is pathogenic.

This mutation results in a radical amino acid substitution at position 1456 in the fourth transmembrane segment (S4) of the fourth repeated domain. ${ }^{7}$ This $\mathrm{S} 4$ segment normally contains a high density of positively charged amino acids (lysine and arginine). The G1456E substitution results in a net alteration of charge from neutral to negative. This leads us to speculate that in keeping with other mutations causing paramyotonia, the present mutation will interfere with voltage sensing and channel inactivation in a temperature dependent fashion. ${ }^{8}$

There are now over 20 different point mutations in the SCN4A gene associated with different phenotypes. The relation between genotype and phenotype is complex and is not always consistent. For example-some point mutations, such as the M1592V mutation causing hyperkalaemic periodic paralysis and the L1433R mutation causing paramyotoniaexhibit a constant genotype-phenotype relation. ${ }^{9}{ }^{10}$ By contrast, other point mutations, such as the R1448C mutation, may cause paramyotonia alone or paramyotonia in combination with hyperkalaeimic periodic paralysis in different families. ${ }^{311}$ The mechanisms underlying this variation in the relation between genotype and phenotype are unclear but the presence of additional modifying genetic factors is one possibility. The finding that the G1456E substitution produces pure paramyotonia in two entirely different genetic backgrounds (Irish and Japanese) may suggest that it will consistently cause pure paramyotonia.

In conclusion, we provided evidence that the G1456E substitution is pathogenic and associated with a pure paramyotonia phenotype. In addition, the present findings confirm that exon 24 in SCN4A is a "hot spot" for mutations causing paramyotonia and we have designed a paramyotonia DNA based diagnostic service accordingly.

Financial support from the Wellcome Trust and the Brain Research Trust is gratefully acknowledged.

\footnotetext{
1 Hanna MG, Wood NW, Kullman DM. Ion channels and neurological disease: DNA based diagnosis is now possible and ion channels may be important in common paroxysmal disorders (editorial). $\mathcal{f}$ Neurol Neurosurg Psychiatry 1998;65:427-31.

2 McClatchey AI, McKenna-Yasek D, Cros D, et al. Novel mutations in families with unusual and variable disorders
} 
of the skeletal muscle sodium channel. Nat Genet

1992;2:148-52.
3 Plassart E, Eymard B, Maurs L, et al. Paramyotonia congenita: genotype to phenotype correlations in two families and report of a new mutation in the sodium channel gene. 7 Neurol Sci 1996;142:126-33.

4 Wagner S, Lerche H, Mitrovic N, et al. A novel sodium channel mutation causing a hyperkalemic paralytic and paramyotonic syndrome with reduced penetrance. Neurology 1997;49:1018-25.

5 Streib EW. Differential diagnosis of myotonic syndromes. Muscle Nerve 1987;10:603-15.

6 Sasaki R, Takano H, Kamakura K, et al. A novel mutation in the gene for the adult skeletal muscle sodium channel alpha-subunit (SCN4A) that causes Paramyotonia Congenita of von Eulenburg. Arch Neurol 1999;56:692-6.
7 George AL Jr, Komisarof J, Kallen RG, et al. Primary structure of the adult human skeletal muscle voltage-dependent sodium channel. Ann Neurol 1992;31:131-7.

8 Lerche H, Mitrovic N, Dubowitz V, et al. Pathophysiology of paramyotonia congenita: the R1448P sodium channel mutation in adult human skeletal muscle. Ann Neurol 1996;39:599-608.

9 Rojas CV, Wang J, Schwartz L, et al. A Met-to-Val mutation in the skeletal muscle sodium channel alpha-subunit in hyperkalemic periodic paralysis. Nature 1991;354:387-9.

10 Ptácek LJ, Gouw L, Kwiecinski H, et al. Sodium channel mutations in paramyotonia congenita and hyperkalemic periodic paralysis. Ann Neurol 1993;33:300-7.

11 Ptácek LJ, George AL Jr, Barchi RL, et al. Mutations in an S4 segment of the adult skeletal muscle sodium channel cause paramyotonia congenita. Neuron 1992;8:891-7.

\section{NEUROLOGICAL STAMP}

\section{Robert Barany (1876-1936)}

Born in Vienna, Barany became a medical doctor in 1900. In 1903, he joined the Viennese Politzer's ear clinic and began his research on the vestibular organ. Barany published his first report in 1906, and in 1909 he was appointed docent at the University of Vienna. In 1915 he was awarded the Nobel Prize in physiology and medicine for his work on the physiology and pathology of the vestibular apparatus. The announcement that he was Nobel Laureate came while he was still a Russian prisoner of war in Merv (Turkestan). Through the intercession of Gunnar Holmgren of Uppsala, the Swedish Red Cross Society, and the King of Sweden, he was released, and the prize was presented to him through diplomatic channels. Barany described for the first time the caloric reaction proceeding from the semicircular canals, in which the endolymph increases in specific gravity with cooling, showing a tendency to seak, whereas with warming the specific gravity decreases and the fluid shows a tendency to rise. This very simply obtained reaction has become the basis for the understanding of some labyrinth diseases. Barany's research provided the neurologist with important clues in the diagnosis of diseases of the central vestibular apparatus, and in its connection with the nucleus of the ocular muscles, the cerebellum, and the spinal cord. After being awarded the Nobel Prize, Barany faced accusations from colleagues of not acknowledging his collaborators' contributions, and he never returned to Vienna. Most of these charges were proved false, but he felt dejected, and spent the rest of his life working in Uppsala, Sweden, where he became Professor of Otorhinolaryngology in 1926. Barany is well known by all ear, nose, and throat specialists because he gave his name to a noise box used in audiological testing. In 1960, his name was adopted by the Barany Society, inaugurated in Padua, Italy, which conducted a vestibular symposium in Uppsala every 5 years.

Barany's involvement with science was best summed up by himself: "The work of a researcher can be divided into three categories: firstly, he needs a sharp and clear perception, must be able to separate the important facts from the less important ones, and must also be able to think independently. Secondly, the researcher must defend his results with all his energy; he must even fight for them. I very often find that important facts are being neglected and even forgotten only because their discoverer did not stand

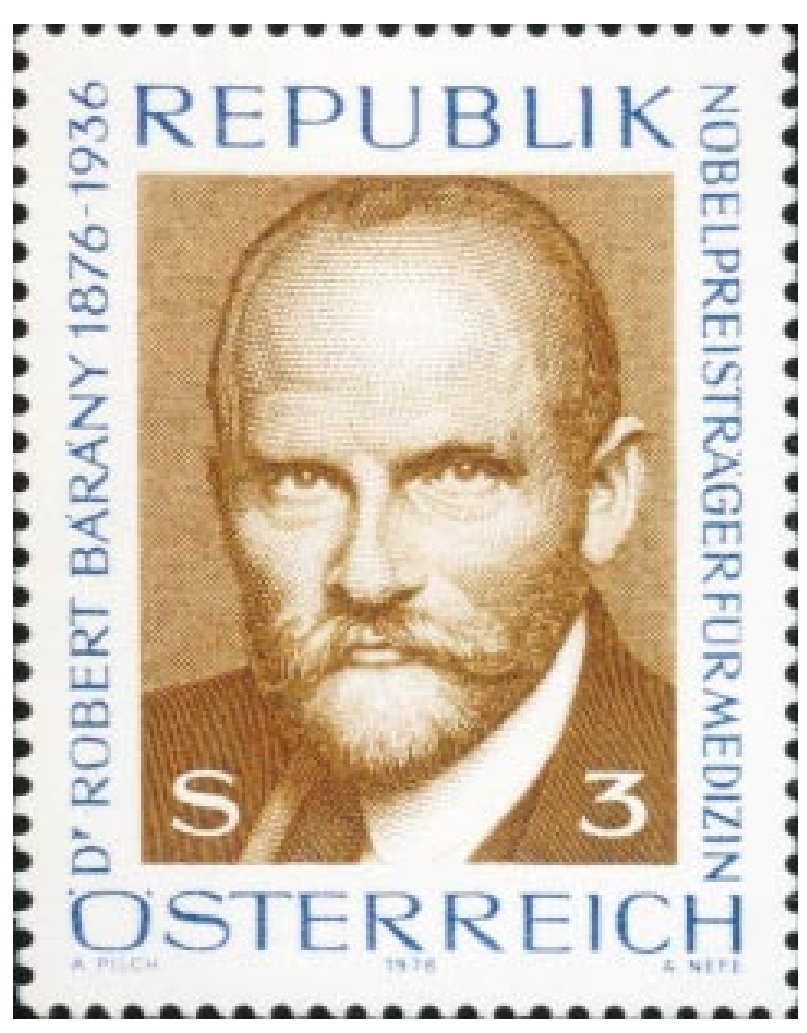

up for them sufficiently. Lastly, the researcher must invest the maximum of his energies into founding his own school of followers, for a single person can only-in his short life span-deal with and solve a fraction of the questions that need to be solved."

In 1976, Austria issued a postage stamp commemorating the 100th anniversary of the birth of Robert Barany. He was also honoured by a Swedish stamp on the 60th anniversary of his Nobel Prize award and by a Hungarian stamp issued in 1988.

ALBERT MUDRY Private practice and ENT Department, University Hospital (CHUV) Lausanne, Avenue de la Gare 6, CH-1003 Lausanne, Switzerland email amudry@worldcom.ch 